\title{
The Problem Optimization Triangular Geometric Line Field
}

\author{
Vasilij Dmitrievich Antoshkin ${ }^{1}$, Vladimir Iljich Travush ${ }^{2}$, Vladimir Trofimovich Erofeev ${ }^{1}$, Vladimir Ivanovich \\ Rimshin $^{3} \&$ Vladimir Leonidovich Kurbatov ${ }^{4}$ \\ ${ }^{1}$ Mordovian State University, Saransk, Russia \\ ${ }^{2}$ RAASN, Moscow, Russia \\ ${ }^{3}$ Director of institute of a housing-and-municipal complex National research Moscow state construction \\ university, Moscow, Russian Federation \\ ${ }^{4}$ The director of North Caucasian branch of the Belgorod state technological university of V.G.Shukhov., \\ Russian Federation \\ Correspondence: Vasilij Dmitrievich Antoshkin, Mordovian State University, Saransk, Russia.
}

Received: October 17, 2014

Accepted: October 27, $2014 \quad$ Online Published: December 11, 2014

doi:10.5539/mas.v9n3p46

URL: http://dx.doi.org/10.5539/mas.v9n3p46

\begin{abstract}
The problems associated with the formation of a geometric network on a sphere. Determining the position of the basic elements of the network to allow the field to optimize network basic criteria. Investigated a method of formation of triangular networks on the sphere. Optimality criteria may be for this the following parameters: minimum number of elements of the dome sizes, the ability to pre-assembly and prestressing.
\end{abstract}

Keywords: team spherical shell, geometric network, panel, a regular hexagon, cutting the dome

\section{Introduction}

The problem of optimizing a triangular geometric network on the sphere by the minimum sizes of elements already becoming the author of various systems of spherical cutting Chebyshev, BV Miryaevym etc. (Alekseevskii, D.V. et al, 1993; Burago, Y. D. et al, 2005; Miryaev, B.V., 2012). In all cases, there was one or more circuits cuttings spheres using mainly a symmetry axis of the main lines, the parallel lines of the cross sections sphere portions compatibility regular polyhedrons faces. Obviously, the placement on the sphere of right and wrong hexagons inscribed, flat shapes or composed in turn of spherical triangles (see Figure 1, b) is the optimal solution in the form of the network formed on the basis of the minimum radii of the circles, ie is circles on a sphere, obtained at the touch of three adjacent circles whose centers are at the shortest distance from each other (Tupolev, M. S., 1969; Travush, V. I. et al, 2013). Spherical quadrilateral with given sides has a maximum when inscribed in a circle. This direction is actively developed by foreign scientists, and by Novosibirsk school in Russia (M'Clelland, W. J. \& Preston, T. A., 1886; Bretschneider, C. A., 1842; Lienhard, W., 2011; Software Scilab). Hexagon panels, inscribed into the circles with the minimum radii will have minimum sizes and squares for given sum of planes triangular network on the sphere. In this case, the elements of the network will have a minimum length, as will be at the shortest distance from the center of the circles, shapes, inscribed in a circle, will also have a minimum size. Education regular hexagons in this network may as a special case. Thus, for each option of cutting, we know the optimal solution for a minimum of material (length elements) - is the placement of a hexagon inscribed in a circle, and in the first version every three adjacent circles touch each other. Each optimization decisions on other criteria will be as follows for the best - "postoptimal" (Annaswamy, A. M. \& Loh, A. P., 1999; Bourbaki N., 1990; Hartskhorn, R., 1970; Alexandrov, P.S., 1975).

\section{The Main Part}

Thus, the optimization of triangular geometric network on the sphere by the minimum sizes of elements can be represented as postoptimization and solved placement in the system wrong hexagons inscribed minimum size of the maximum regular hexagons, for example, in compatible spherical triangles (segments) of the sphere with circuits cutting shown in Figure 1a, b. Also applications of the symmetry properties of parallel lines and the main areas where such cutting opportunities should be implemented central symmetry circles (Banga, S. et al., 2014; Burdette, A. C., 1971). On the diagram of Figure 1 shows the placement of the circumscribed circle of hexagons in a spherical triangle (compatible segment of the sphere) with interior angles 36,90 and $60^{\circ}$. 


\subsection{Definition of Position of the Centers of Circles of Hexagons}

When optimizing a triangular network using symmetry properties of circles and spheres of the main lines can be identified as one of the tasks - determination of the centers of the circles hexagons (Figures $1 \mathrm{a}, \mathrm{b}$ : the centers of the first rows of hexagons $\mathrm{O}_{0}, \mathrm{O}_{1}, \mathrm{O}_{2}$ ). In Figure 1 it is shown how to correct spherical hexagon (right triangle) the largest radius is located on the axis of symmetry as the smallest angle bisectors of any triangle on the sphere. On this basis, we determine the position of the center $\mathrm{O}$ of a regular hexagon in the spherical triangle with internal angles A, B, C.

Compose using relations Napier (Granino, A., 1968), the system of equations (1)

$$
\begin{gathered}
x=e-y, \\
\sin d=\sin z / \sin A, \\
\sin z=\sin x \sin 60^{\circ},(1) \\
\cos d=\cos x \cos y+\sin x \sin y \cos 60^{\circ},
\end{gathered}
$$

where: $e$ - bisector arc from vertex A to a polar angle of arbitrary spherical triangle with acute angles (see Figure $1) ; x$ - radius of the circle describing the regular hexagon (shown in the problem statement in the figure as a spherical equilateral triangle) as the polar angle; $y$ - an arc from vertex A to the center of the circle circumscribing the equilateral triangle as the polar angle; $z$ - this side of the equilateral triangle as the polar angle; $\mathrm{A}, \mathrm{B}$ and $\mathrm{C}$ are arbitrary spherical triangle vertices; A and D, respectively, the interior angles in any spherical triangle on a sphere.

\subsection{Elementary Transformations}

Through Elementary Transformations, We Obtain:

$$
\begin{gathered}
1-\left(\frac{\sin x \sin 60^{\circ}}{\sin A}\right)^{2}=\left(\cos x \cos y+\cos 60^{\circ} \sin x \sin y\right)^{2}, \\
\sin ^{2} A-\frac{3}{4} \sin ^{2} x=\left(\cos ^{2} x \cos ^{2} y+\cos x \cos y \sin x \sin y+\frac{1}{4} \sin ^{2} x \sin ^{2} y\right) \sin ^{2} A . \\
\frac{3}{4}\left(\sin ^{2} A-1\right) \operatorname{tg}^{2} x \operatorname{tg}^{2} y+\left(\sin ^{2} A-\frac{3}{4}\right) \operatorname{tg}^{2} x+\sin ^{2} A \operatorname{tg}^{2} y-\sin ^{2} A \operatorname{tg} x \operatorname{tg} y=0 .
\end{gathered}
$$

Denote half the interior angles for vertex $\mathrm{A}$ as $A, \operatorname{arc} e$ as polar angle $E A=e=x+y, \operatorname{arc} F A=d$. Given that $x=$ $e y$, and introducing the notation: $k_{e}=\operatorname{tg} e, t=\operatorname{tg} y$, we obtain

Turning to equation (4), we obtain:

$$
\operatorname{tg} x=\frac{k_{e}-t}{1+k_{e} t}
$$

$$
\frac{3}{4}\left(\sin ^{2} A-1\right)\left(\frac{k_{e}-t}{1+k_{e} t}\right)^{2} t^{2}+\left(\sin ^{2} A-\frac{3}{4}\right)\left(\frac{k_{e}-t}{1+k_{e} t}\right)^{2}++\sin ^{2} A t^{2}-\sin ^{2} A \frac{k_{e}-t}{1+k_{e} t} t=0 .
$$

Multiply equation (5) $\left(1+k_{e} t\right)^{2} \neq 0$ and, after elementary transformations, webobtain:

$$
\begin{gathered}
\left(k_{e}{ }^{2} \sin ^{2} A-\frac{3}{4} \cos ^{2} A\right) t^{4}+3 k_{e}\left(\sin ^{2} A+\frac{1}{2} \cos ^{2} A\right) t^{3}+\left(\left(3-k_{e}{ }^{2}\right) \sin ^{2} A-\frac{3}{4} k_{e}{ }^{2} \cos ^{2} A-\frac{3}{4}\right) t^{2}+3 k_{e}\left(\frac{1}{2}-\right. \\
\left.\sin ^{2} A\right) t+k_{e}{ }^{2}\left(\sin ^{2} A-\frac{3}{4}\right)=0 .
\end{gathered}
$$




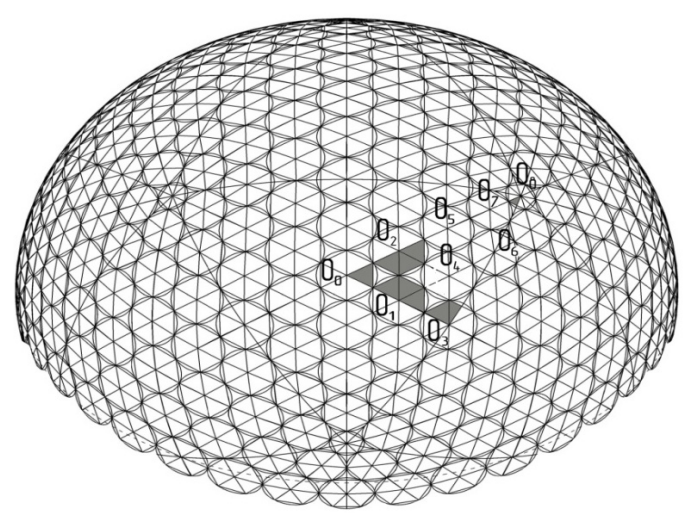

a)

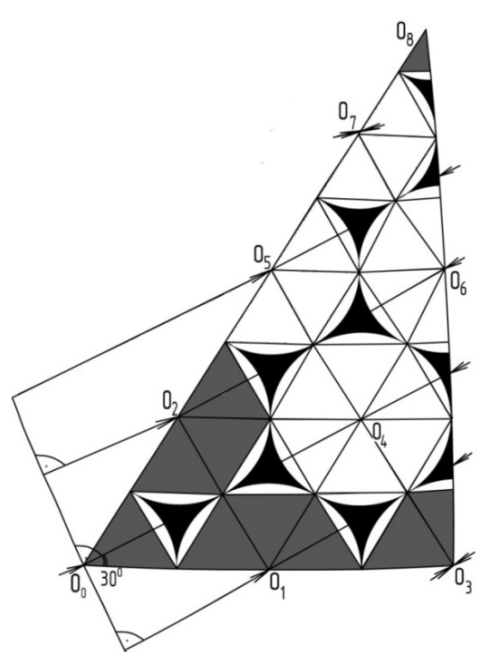

b)

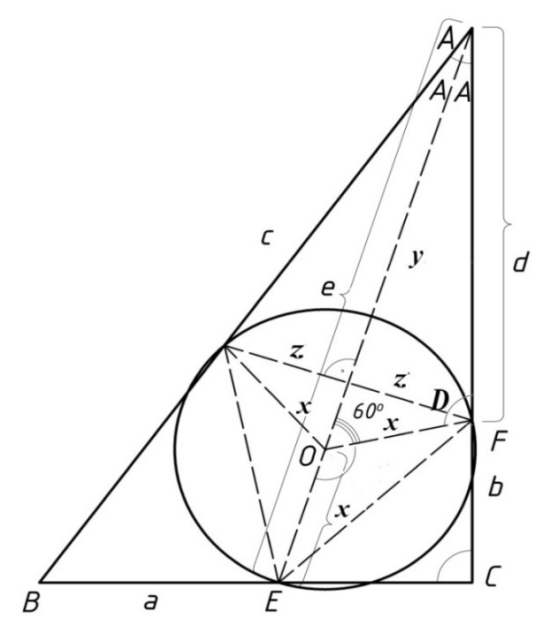

c)

Figure 1. Layouts flat or spherical hexagons in a triangular network optimization problems using the symmetry properties of circles and spheres of the main lines (shown in gray regular polygons) and - hemisphere by cutting a triangular network in 3840 as a polyhedron composed of compatible spherical triangles (segments representing the sixth face of the icosahedron); $b$ - the layout of the flat hexagonal segment with internal angles $36,90,60$ degrees in solving the optimization problem of the triangular network using the symmetry properties of circles and spheres of the main lines (main lines are axes within a spherical segment); $\mathrm{c}$ - circuit for determining the position of the center $\mathrm{O}$ of the minimum radius of a regular hexagon (or equilateral triangle) in the spherical triangle with arbitrary internal angles $A, B, C$

Divide both sides of equation (6) to $\cos ^{2} A$, and arrive at the dependence

$$
\left(k_{e}{ }^{2} \operatorname{tg}^{2} A-\frac{3}{4}\right) t^{4}+3 k_{e}\left(\operatorname{tg}^{2} A+\frac{1}{2}\right) t^{3}+\left(\left(\frac{9}{4}-k_{e}{ }^{2}\right) \operatorname{tg}^{2} A-\frac{3}{4}\left(k_{e}{ }^{2}+1\right)\right) t^{2}+\frac{3}{2} k_{e}\left(1-\operatorname{tg}^{2} A\right) t+\frac{1}{4} k_{e}{ }^{2}\left(\operatorname{tg}^{2} A-3\right)=0 ;
$$

Finally, by introducing in equation (7), the coefficient $k_{A}=\operatorname{tg} A$, arrive at the desired equation

$$
\left(k_{e}{ }^{2} k_{A}{ }^{2}-\frac{3}{4}\right) t^{4}+3 k_{e}\left(k_{A}{ }^{2}+\frac{1}{2}\right) t^{3}+\left(\left(\frac{9}{4}-k_{e}{ }^{2}\right) k_{A}{ }^{2}-\frac{3}{4}\left(k_{e}{ }^{2}+1\right)\right) t^{2}+\frac{3}{2} k_{e}\left(1-k_{A}{ }^{2}\right) t+\frac{1}{4} k_{e}{ }^{2}\left(k_{A}{ }^{2}-3\right)=0 .
$$

Apply the results to solve the problem in the case of an isosceles triangle with an angle $2 A=60^{\circ}$. Determine the bisector on the specified sides of a spherical triangle. Consider a triangle with the bisector of equal

$$
\mathrm{e}=20.90516288636^{\circ} \text {. }
$$


Then the estimated coefficients, the following expression:

$$
k_{e}=\operatorname{tg} e=0.3819661, k_{A}=\operatorname{tg} A=0.5773503 .
$$

Thus, equation (8) takes the form

$$
0.7013673 t^{4}-0.9549153 t^{3}+0,1580563 t^{2}-0,3819661 t+0,0972654=0 .
$$

Conditions of the problem, the root of the equation $\mathrm{t}=0,02487930$ satisfies problem where we find $\mathrm{y}=$ $13,971137^{\circ}$. Then determined the radius and position of the center of a regular hexagon minimum radius $\mathrm{x}=$ $6,9340259^{\circ}$. To solve the problem use the freely available software Scilab 5.4.1 - The free platform for Numerical Computation (analogue Matlab) (Analytical Geometry., 1963; Advanced Calculus with Linear Analysis., 1972; Advanced Calculus and Vector Field Theory., 1966).

\section{Findings}

The resulting solutions will realize the scope of approximation algorithms triangular geometric network with the maximum number of regular hexagons and prepare options for optimizing section sphere.

\section{References}

Advanced Calculus and Vector Field Theory. (1966). pp. 263-265. http://dx.doi.org/10.1016/B978-1-4831-6785-5.50014-7

Advanced Calculus with Linear Analysis. (1972). http://dx.doi.org/10.1016/B978-0-12-440750-3.50001-7

Alekseevskii, D. V., Vinberg, E. B., \& Solodovnikov, A. S. (1993). Geometry of spaces of constant curvature. Results in science and technology. Current problems in mathematics. Fundamental Directions. VINITI, 29, $1-146$.

Alexandrov, P. S. (1975). Introduction to the homological theory of dimension and general combinatory topology. (pp. 139-148).

Analytical Geometry. (1963). A volume in The Commonwealth and International Library of Science, Technology, Engineering and Liberal Studies: Mathematics Division, 46-64. http://dx.doi.org/10.1016/B978-0-08-009973-6.50001-1

Annaswamy, A. M., \& Loh, A. P. (1999). Stable adaptive systems in the presence of nonlinear parametrization. Adaptive Control Systems, 215-259. http://dx.doi.org/10.1016/B978-075063996-5/50010-5

Banga, S., Dubickasb, A., Koolenc, J. H., \& Moultond, V. (2014). There are only finitely many distance-regular graphs of fixed valency greater than two. Advances in Mathematics, 269, 1-55. http://dx.doi.org/10.1016/j.aim.2014.09.025

Bourbaki, N. (1990). Mathematical analysis and geometry, 45, 94-97.

Bretschneider, C. A. (1842). Unetersuchung der trigonometrischen Relationen des geradlinigen Viereckes. Arch. Math. Bd, 2, 225-261.

Burago, Y. D., Ivanov, S. V., \& Malev, S. G. (2005). Remarks on Chebyshev coordinates. Geometry and topology, 5-13. St. Petersburg.

Burdette, A. C. (1971). Analytic Geometry (pp. 165-176). http://dx.doi.org/10.1016/ B978-0-12-142256-1.50001-2

Granino, A. (1968). Korn Mathematical handbook: For scientists and engineers.

Hartskhorn, R. (1970). Fundamentals of projective geometry (pp. 45-53).

Lienhard, W. (2011). Cyclic polygons in non-Euclidean geometry. Elem. math., 66(2), 74-82.

M'Clelland, W. J., \& Preston, T. A. (1886). treatise on spherical trigonometry with application to spherical geometry and numerous examples. P. II. London: Macmillian and Co.

Miryaev, B. V. (2012). Optimization of the geometry of reticulated domes on the basis of ikosahedron. Regional architecture and engineering, 3, 122-125.

Software Scilab 5.4.1 The free platform for Numerical Computation. Retrieved June 17, 2014, from Retrieved from www.softkumir.ru/index

Travush, V. I., Antoshkin, V. D., Erofeev, V. T. Team spherical shell. Patent RU №2012116363 from 20.02.14 year.

Travush, V. I., Antoshkin, V. D., Erofeev, V. T. Team spherical shell. A utility model patent RU № 129534 from 
06.27.13 year.

Tupolev, M. S. (1969). Geometry prefabricated spherical domes. Architecture of the USSR, 1, 15-17.

\section{Copyrights}

Copyright for this article is retained by the author(s), with first publication rights granted to the journal.

This is an open-access article distributed under the terms and conditions of the Creative Commons Attribution license (http://creativecommons.org/licenses/by/3.0/). 